\title{
0114 DISTRIBUTION OF INJURY DEATH AND OUTPATIENTS IN RURAL AND URBAN AREAS OF CHINA IN 2006
}

L Duan*, X Deng Correspondence: National Center for Chronic and Noncommunicable Disease Control and Prevention, China CDC, No 27, Nan Wei Road, Xuan Wu District, Beijing, P. R. China, 100050, China

10.1136/ip.2010.029215.114

Objectives To know the distribution features of injury of Chinese population in rural and urban areas, and further to provide basic scientific data for injury prevention and control in different areas.

Methods Fatal injury data were derived from official death certificates by the National Disease Surveillance Points System (DSP). Hospital injury visit data were from emergency and outpatient rooms of the 126 hospitals of the National Injury Surveillance System (NISS). Descriptive analysis was used to explore the differences between and similarities in rural and urban areas.

Results While the order of the five main causes of injury deaths were the same in rural and urban areas, mortalities of injury and main types of injury were higher in rural areas than urban areas in 2006. Most of the hospital injury visits from emergency and outpatient rooms were male, 15-64 years old both in rural and urban areas in 2006. The cause 


\section{IP Safety 2010 abstracts}

or mechanism, location, activity and intent of injury and the clinical conditions of injury patients have both differences and similarities in rural and urban areas.

Conclusion The epidemiologies of both death and hospital visits from injury are different between rural and urban areas in China according to the national surveillance systems. Thus, appropriate strategies and schemes should be taken in injury prevention and control in different areas. 